\title{
Research on the Metal's Three-dimentional Plastic Flow Mechanism in Rail Universal Rolling
}

\author{
Yong-Gang DONG ${ }^{1, a,{ }^{*}}$, Guo-Ling LUO ${ }^{1, b}$, Zhong-Chao REN ${ }^{1, c}$ \\ ${ }^{1}$ College of mechanical engineering, Yanshan University, Qinhuangdao,China \\ ad_peter@163.com, b1475116436@qq.com, 'c1434309470@qq.com \\ ${ }^{*}$ Corresponding author
}

Keywords: Rail, Universal Rolling, Plastic Flow, Elongation Coefficient, Flow Volume.

\begin{abstract}
For studying the metal's three-dimentional platic flow mechanism along the longitudinal direction and lateral direction in rail universal rolling, the relationship between the metal flow volume and the elongation coefficient of different parts of rail was proposed, then the elongation coefficient of different parts of rail were derived on the base of the law of volume constancy, then the flow volume between different parts of rail were obtained. Moreover, the integrated deformation influence factor was introduced into this model to express the influence of the spread coefficient and the reduction coefficient of different parts of rail on the metal's three-dimentional plastic flow. Compared with the experimental results, the prediction error of analytical model for elongation coefficient is less than $\pm 5 \%$, and the the prediction error of analytical model for flow volume is not greater than $\pm 16 \%$.
\end{abstract}

\section{Introduction}

The universal rolling method has been applied in rail rolling for 40 years. At present, the process of the rail universal rolling has been studied by FEM and experimental methods generally. In the Reference $^{[1-3]}$, the process of rail universal rolling was studied by FEM software and the rolling experiments, then the stress distribution and deformation law of rail universal rolling were analyzed. In the Reference ${ }^{[5]}$, the spread model of the base and head of rail in rail rough rolling was proposed on the base of existing empirical functions. In the Reference ${ }^{[6,7]}$, the spread and elongation law of heavy rail universal rolling were analyzed by the nerve network method based on a number of measured data.

As mentioned above, these analytic model for calculating the spread and elongation of rail universal rolling were all derived by the empirical equation directly or indirectly, and the metal's lateral flow between the base of rail, the head of rail and the waist of rail were neglected. However the volume of metal's lateral flow will influence the cross-section profile of outgoing workpiece and the elongation coefficient of the base of rail, the head of rail and the waist of rail. Therefore, the elongation coefficient and the lateral flow of different parts of rail interrelate with each other.

Consequently, if an accurate mathematical model can be built to predict the volume of metal's lateral flow and the elongation coefficient of different parts of rail, a rational rolling schedule and processing parameters for rail universal rolling could be preset to improve the cross-section size precision and decrease the degree of curvature and the residual stress of finished rail effectively. So the performance of rail can be improved greatly based on a precise mathematical model of three-dimensional plastic flow.

\section{Building the Mathematical Model of Plastic Flow in Rail Universal Rolling}

There are significant differences between the pass profile and cross-section profile of the base, head and waist of rail, and the deformation mechanism of different parts of rail are quite distinct. So the elongation coefficient of the base, head and waist of rail are different generally, there are metal's lateral flow existing in different parts of rail to reduce the discrepancy of elongation coefficient for 
keeping the deformation's compatibility and consistency.

The following equations can be proposed according to the volume constancy .

$$
\left\{\begin{array}{l}
V_{t 1}=V_{t 0}-\Delta V_{t w} \\
V_{b 1}=V_{b 0}-\Delta V_{b w} \\
V_{w 1}=V_{w 0}+\Delta V_{t w}+\Delta V_{b w}
\end{array}\right.
$$

Where $\Delta V_{t w}$ and $\Delta V_{b w}$ are the metal volume flowing from the head of rail and the base of rail to the waist of rail along the lateral direction respectively. $V_{t 0}, V_{b 0}, V_{w 0}$ are the volume of incoming workpiece of the base, head and waist of rail individually, and $V_{t 1}, V_{b 1}, V_{w 1}$ are the volume of outgoing workpiece of the base, head and waist of rail individually.

Applying the model mentioned in reference ${ }^{[8]}$ into the process of rail universal rolling and the volume of metal's lateral flow in rail universal rolling can be expressed by

$$
\left\{\begin{array}{l}
\Delta V_{t w}=\alpha V_{w 1} \ln \left(\lambda_{t} / \lambda_{w}\right) \\
\Delta V_{b w}=\alpha V_{w 1} \ln \left(\lambda_{b} / \lambda_{w}\right)
\end{array}\right.
$$

Where $\alpha$ is a emperical coefficient determined by the experiments, it is defined as 0.6 , and $\lambda_{t}, \lambda_{b}, \lambda_{w}$ are the elongation coefficient of the base of rail, the head of rail and the waist of rail individually.

For reducing the solving difficulty, the Eq.2 can be simplified and shown as

$$
\left\{\begin{array}{l}
\Delta V_{t w}=\alpha V_{w 1}\left(\lambda_{t} / \lambda_{w}-1\right) \\
\Delta V_{b w}=\alpha V_{w 1}\left(\lambda_{b} / \lambda_{w}-1\right)
\end{array}\right.
$$

Substituting Eq. 3 into Eq. 1 and solving it, $\lambda_{t}, \lambda_{b}, \lambda_{w}$ can be expressed individually as

$$
\begin{aligned}
& \lambda_{w}=\frac{T}{\alpha S_{w o} B} \eta_{w}=f\left(\beta_{t} \frac{\eta_{w}}{\eta_{t}}, \beta_{b} \frac{\eta_{w}}{\eta_{b}}, \eta_{w}\right) \\
& \lambda_{t}=\frac{B S_{t 0}+T}{B\left(\alpha S_{w 0}+S_{t 0} \beta_{t}^{*} \eta_{w} / \eta_{t}\right)} \eta_{w}=f\left(\beta_{t} \frac{\eta_{w}}{\eta_{t}}, \beta_{b} \frac{\eta_{w}}{\eta_{b}}, \eta_{w}\right) \\
& \lambda_{b}=\frac{B S_{b 0}+T}{B\left(\alpha S_{w 0}+S_{b 0} \beta_{b} * \eta_{w} / \eta_{b}\right)} \eta_{w}=f\left(\beta_{t} \frac{\eta_{w}}{\eta_{t}}, \beta_{b} \frac{\eta_{w}}{\eta_{b}}, \eta_{w}\right)
\end{aligned}
$$

In Eq.4 Eq.6, parameter $B$ and $T$ can be obtained by

$$
\begin{aligned}
& B=\frac{2 \alpha+1}{\alpha}-\frac{\alpha}{\frac{S_{t 0}}{S_{w 0}} \beta_{t} \frac{\eta_{w}}{\eta_{t}}+\alpha}-\frac{\alpha}{\frac{S_{b 0}}{S_{w 0}} \beta_{b} \frac{\eta_{w}}{\eta_{b}}+\alpha} \\
& T=\frac{\alpha S_{w 0}}{\beta_{t} \frac{\eta_{w}}{\eta_{t}}+\frac{\alpha}{V_{t 0}}}+\frac{\alpha S_{w 0}}{\beta_{b} \frac{\eta_{w}}{\eta_{b}}+\frac{\alpha}{V_{b 0}}}+S_{w 0}
\end{aligned}
$$

Where $\eta_{t}, \eta_{b}, \eta_{w}$ are the reduction coefficient of the base, the head and the waist of rail individually, $\beta_{t}, \beta_{b}$ are the spread coefficient of the base the head of rail individually which can be obtained in reference $^{[9,10]}$, the spread coefficient of the waist of rail $\beta_{w}$ is 1.0 for the width of the wasit of rail is considered as a constant. The factor $\beta_{t} * \eta_{w} / \eta_{t}$ and $\beta_{b} * \eta_{w} / \eta_{b}$ are defined as integrated deformation influencing factors.

$\Delta V_{t w}, \Delta V_{b w}$ can be obtained according to the Eq.3. 


$$
\begin{aligned}
& \Delta V_{t w}=\alpha V_{w 0}\left(\frac{V_{t 0}}{\left(\frac{\eta_{w}}{\eta_{t}} \beta_{t}\right) V_{t 0}+\alpha V_{w 0}}+\frac{\lambda_{w}}{\eta_{w}}\left(\frac{\alpha V_{w 0}}{\left(\frac{\eta_{w}}{\eta_{t}} \beta_{t}\right) V_{t 0}+\alpha V_{w 0}}-1\right)=f\left(\beta_{t} \frac{\eta_{w}}{\eta_{t}}, \beta_{b} \frac{\eta_{w}}{\eta_{b}}\right)\right. \\
& \Delta V_{b w}=\alpha V_{w 0}\left(\frac{V_{b 0}}{\left(\frac{\eta_{w}}{\eta_{b}} \beta_{b}\right) V_{b 0}+\alpha V_{w 0}}+\frac{\lambda_{w}}{\eta_{w}}\left(\frac{\alpha V_{w 0}}{\left(\frac{\eta_{w}}{\eta_{b}} \beta_{b}\right) V_{b 0}+\alpha V_{w 0}}-1\right)\right)=f\left(\beta_{t} \frac{\eta_{w}}{\eta_{t}}, \beta_{b} \frac{\eta_{w}}{\eta_{b}}\right)
\end{aligned}
$$

As shown as Eq.9 and Eq.10, the flow volume $\Delta V_{b w}$ and $\Delta V_{b w}$ are only influenced by the integrated deformation influencing factors $\beta_{t}^{*} \eta_{w} / \eta_{t}$ and $\beta_{b} * \eta_{w} / \eta_{b}$.

\section{Calculating Results of the Elongation and the Metal's Volume Flow}

According to Eq.4 Eq.6, by the Software Matalab 7.0, the elongation coefficient and the volume of metal's lateral flow were solved, and they are all related to $\beta_{t} * \eta_{w} / \eta_{t}, \beta_{b} * \eta_{w} / \eta_{b}$ and $\eta_{w}$. Calculating results of the elongation coefficient were shown as Fig.1 Fig.5

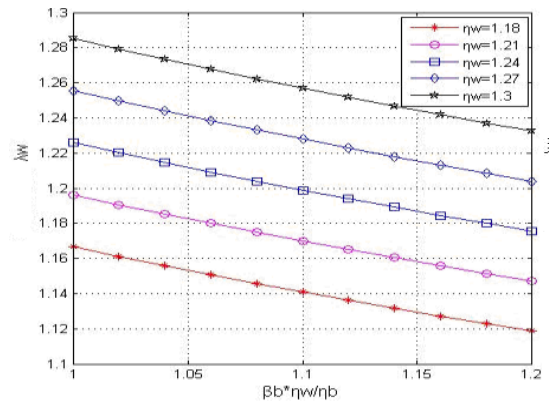

(a) $\beta_{t} * \eta_{w} / \eta_{t}=1.05$

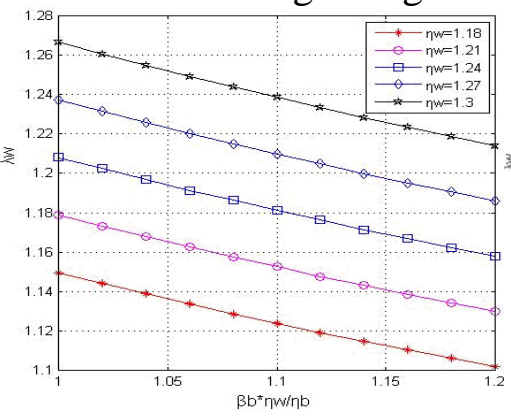

(b) $\beta_{t}^{*} \eta_{w} / \eta_{t}=1.12$

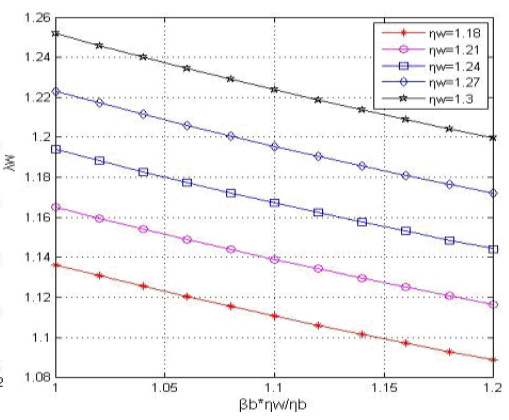

(c) $\beta_{t}^{*} \eta_{w} / \eta_{t}=1.18$

Fig.1 Curve of Elongation Coefficien of the Waist of Rail $\lambda_{w}$

As shown in Fig.1, if $\eta_{w}$ is known, the elongation coefficient of the waist of rail decreases with the increment of $\beta_{b} * \eta_{w} / \eta_{b}$ and $\beta_{t} * \eta_{w} / \eta_{t}$. Moreover, if the deformation coefficient of the base and head of rail are determined, the elongation coefficient of the waist of rail increases with the increment of the reduction coefficient.

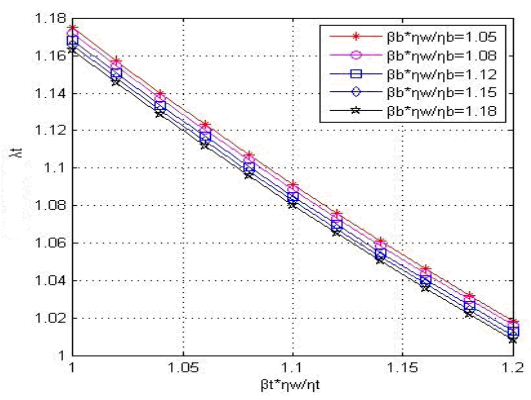

(a) $\eta_{w}=1.18$

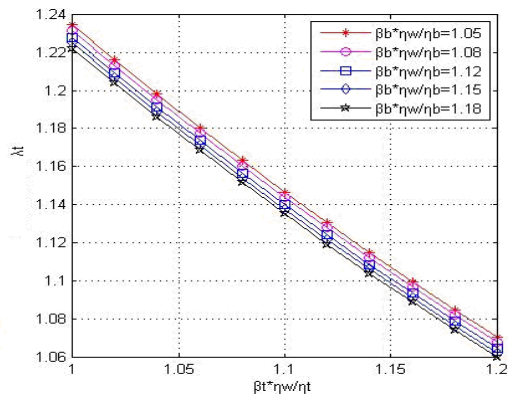

(b) $\eta_{w}=1.24$

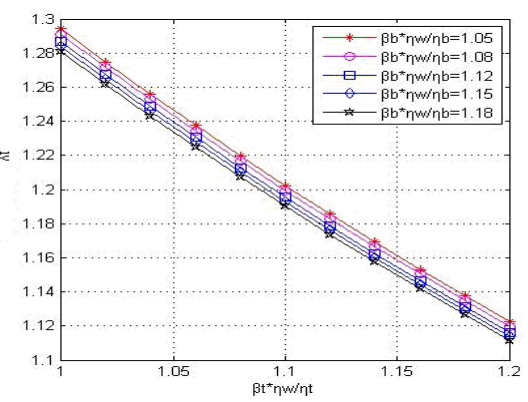

(c) $\eta_{w}=1.30$

Fig. 2 Curve of Elongation Coefficien ${ }^{\lambda_{t}}$ of the Top of Rail

As shown in Fig.2, based on the same condition of deformation parameters, the variation tendency of the elongation coefficient of the head of rail is in accord with that of the elongation coefficient of the base of rail. The elongation coefficient of the head of rail doesn't vary with $\beta_{b} * \eta_{w} / \eta_{b}$ sharply. That's to say, the deformation of the base of rail does not influence the elongtation coefficient of the head of rail obviously. 


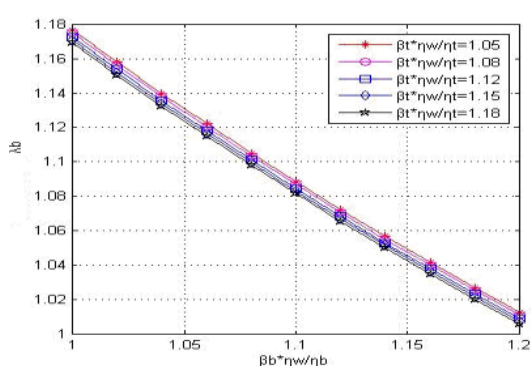

(a) $\eta_{w}=1.18$

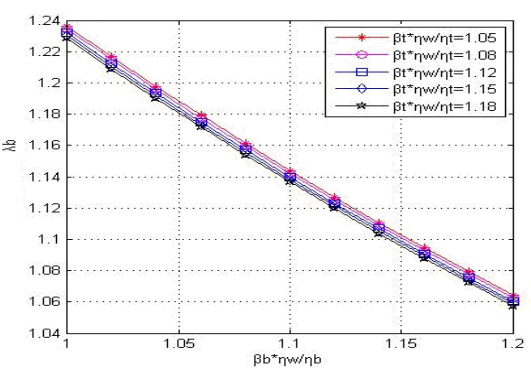

(b) $\eta_{w}=1.24$

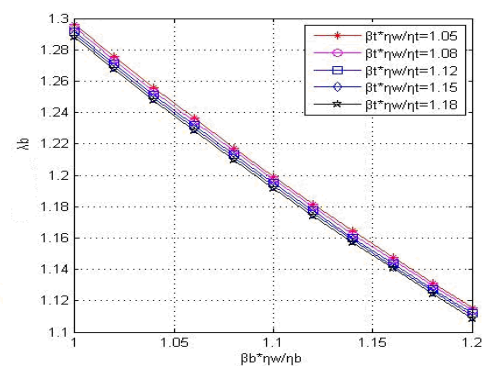

(c) $\eta_{w}=1.30$

Fig. 3 Curve of Elongation Coefficien of the Base of Rail $\lambda_{b}$

As shown in Fig.3, if the other deformation parameters are given, the elongation coefficient of the base of rail increases with the increment of $\eta_{w}$. However, the elongation coefficient of the base of rail decreases with the increment of $\beta_{b} * \eta_{w} / \eta_{b}$ obviously. Moreover, the elongation coefficient of the base of rail doesn't vary with $\beta_{t}^{*} \eta_{w} / \eta_{t}$ sharply.

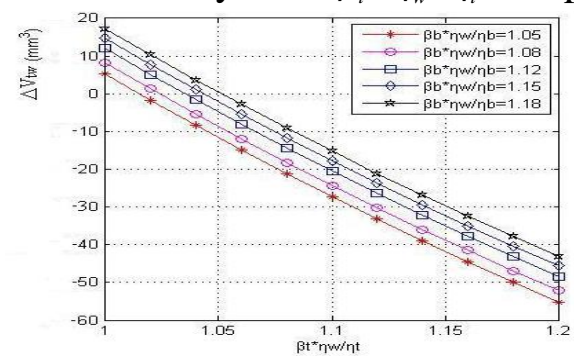

Fig.4 Curve of Flow Volume $\Delta V_{t w}$

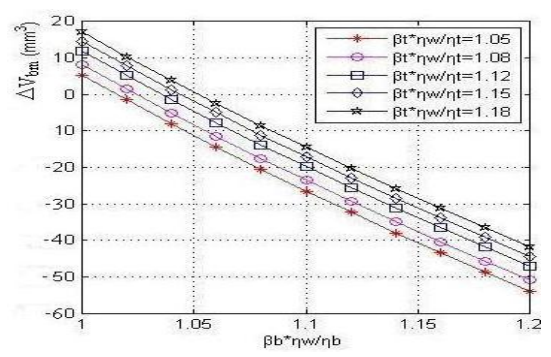

Fig.5 Curve of Flow Volume $\Delta V_{b w}$

As shown in Fig.4 and Fig.5, with the rise of $\beta_{t}^{*} \eta_{w} / \eta_{t}$ and $\beta_{b}{ }^{*} \eta_{w} / \eta_{b}$, the quatity and direction of volume flow between different parts of rail changes obviously.

\section{Rolling Experiments and Its Results}

For verifying results from the theoretical model, rolling experiments was accomplished in Yanshan university. In experiments the three-stand universal mill and box-type heater were used. A piece of $18 \mathrm{~kg} / \mathrm{m}$ light rail was purchased and it was separated into 18 portions and divided into 6 groups, and 3 portions was a group and the length of every portion was $300 \mathrm{~mm}$. The cross-section size of the $18 \mathrm{~kg} / \mathrm{m}$ light rail was shown as Fig. 6.

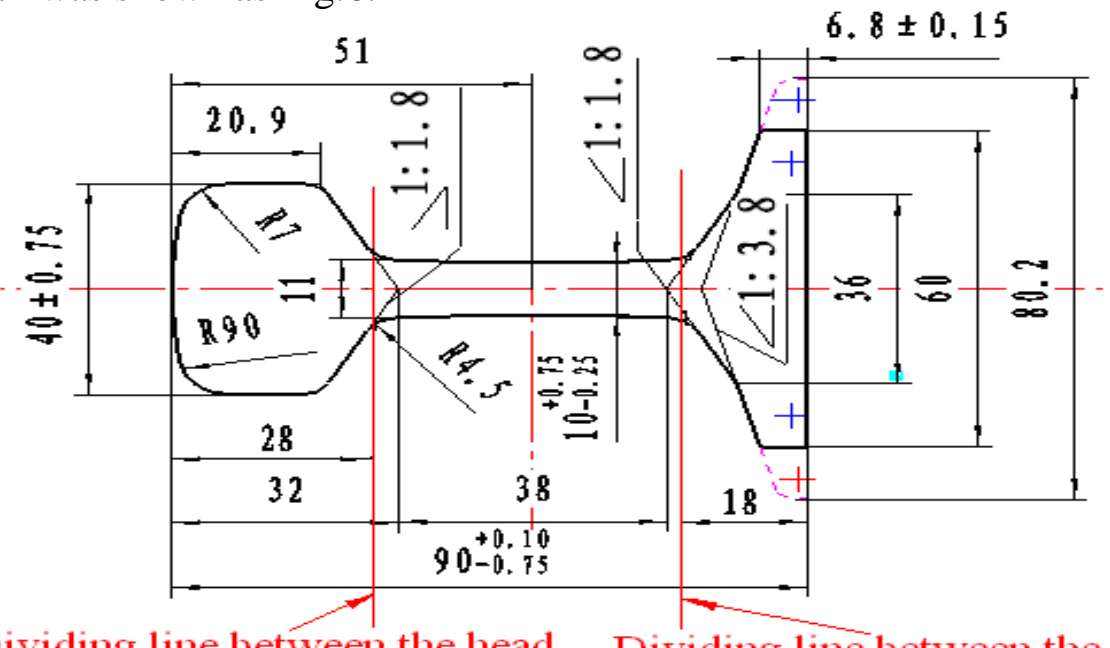

Dividing line between the head of rail and the waist of rail

Dividing line between the base of rail and the waist of rail

Fig.6 Cross-section Size of Initial Structural Section 
Workpieces of every group was rolled with a different processing technology by the universal mill with three stands, and the rolling schedule was expressed as Table 1 . The cross-section size and the volume of the base of rail, the head of rail and the waist of rail can be obtained by the mean value of measured results of three workpieces in a same group.

Tab.1 Rolling Schedule for Universal Rough Stand in Hot Rolling Experiments

\begin{tabular}{ccccc}
\hline \multirow{2}{*}{ Group No. } & \multirow{2}{*}{ Workpiece No. } & \multicolumn{3}{c}{ Reduction coefficient(mm) } \\
\cline { 2 - 5 } & & $\eta_{w}$ & $\eta_{t}$ & $\eta_{b}$ \\
\hline 1 & $1-11-21-3$ & 1.1765 & 1.1905 & 1.25 \\
\hline 2 & $2-12-22-3$ & 1.1765 & 1.2195 & 1.25 \\
\hline 3 & $3-13-23-3$ & 1.2500 & 1.1628 & 1.25 \\
\hline 4 & $4-14-24-3$ & 1.2500 & 1.1364 & 1.25 \\
\hline 5 & $5-15-25-3$ & 1.3333 & 1.1111 & 1.25 \\
\hline 6 & $6-16-26-3$ & 1.3333 & 1.1111 & 1.25 \\
\hline
\end{tabular}

As shown in Table 2, analytical results of elongation coefficient $\lambda_{t}, \lambda_{b}$ and $\lambda_{w}$ were compared with that of measured results. The prediction error of elongation coefficient is less than $5 \%$.

Tab.2 The Elongation Ratio of the Rail in Universal Rolling

\begin{tabular}{|c|c|c|c|c|c|c|c|c|c|}
\hline & \multicolumn{3}{|c|}{ Elongation coefficient $\lambda_{t}$} & \multicolumn{3}{|c|}{ Elongation coefficient $\lambda_{w}$} & \multicolumn{3}{|c|}{ Elongation coefficient $\lambda_{b}$} \\
\hline $\begin{array}{c}\text { Group } \\
\text { No. }\end{array}$ & $\begin{array}{c}\text { Measured } \\
\text { Resluts }\end{array}$ & $\begin{array}{c}\text { Analytical } \\
\text { Resluts }\end{array}$ & Error & $\begin{array}{c}\text { Measured } \\
\text { Resluts }\end{array}$ & $\begin{array}{c}\text { Analytical } \\
\text { Resluts }\end{array}$ & Error ${ }^{1}$ & $\begin{array}{c}\text { Measurec } \\
\text { Resluts }\end{array}$ & $\begin{array}{c}\text { Analytical } \\
\text { Resluts }\end{array}$ & Error \\
\hline 1 & 1.19 & 1.173 & $-1.4 \%$ & 1.17 & 1.227 & $+4.9 \%$ & 1.18 & 1.219 & $+1.4 \%$ \\
\hline 2 & 1.21 & 1.190 & $-1.7 \%$ & 1.19 & 1.246 & $+4.7 \%$ & 1.20 & 1.230 & $+2.5 \%$ \\
\hline 3 & 1.15 & 1.156 & $+0.4 \%$ & 1.23 & 1.285 & $+4.5 \%$ & 1.22 & 1.247 & $+2.2 \%$ \\
\hline 4 & 1.14 & 1.128 & $-1.1 \%$ & 1.21 & 1.266 & $+4.6 \%$ & 1.20 & 1.232 & $+2.7 \%$ \\
\hline 5 & 1.09 & 1.137 & $+4.3 \%$ & 1.25 & 1.305 & $+4.4 \%$ & 1.24 & 1.256 & $+1.3 \%$ \\
\hline 6 & 1.08 & 1.117 & $+3.4 \%$ & 1.28 & 1.318 & $+3.0 \%$ & 1.24 & 1.264 & $+1.9 \%$ \\
\hline
\end{tabular}

Tab.3 Flow Volume between Different Parts of Rail

\begin{tabular}{|c|c|c|c|c|c|c|}
\hline & \multicolumn{2}{|c|}{ Flow volume $\Delta V_{t w}\left(\mathrm{~mm}^{3}\right)$} & \multicolumn{3}{c|}{ Flow volume $\Delta V_{b w}\left(\mathrm{~mm}^{3}\right)$} \\
\hline N0. & $\begin{array}{c}\text { Measured } \\
\text { Resluts }\end{array}$ & $\begin{array}{c}\text { Analytical } \\
\text { Resluts }\end{array}$ & Error & $\begin{array}{c}\text { Measured } \\
\text { Resluts }\end{array}$ & $\begin{array}{c}\text { Analytical } \\
\text { Resluts }\end{array}$ & Error \\
\hline 1 & -20.2 & -22.8 & $-12.87 \%$ & +9.1 & +10.5 & $+15.38 \%$ \\
\hline 2 & -11.8 & -13.6 & $-15.25 \%$ & +7.8 & +8.8 & $+12.82 \%$ \\
\hline 3 & -29.8 & -32.3 & $-8.39 \%$ & -8.9 & -10.1 & $-13.48 \%$ \\
\hline 4 & -34.2 & -38.6 & $-12.87 \%$ & -8.5 & -9.7 & $-14.12 \%$ \\
\hline 5 & -43.7 & -47.8 & $-9.38 \%$ & -17.3 & -19.6 & $-13.29 \%$ \\
\hline 6 & -44.1 & -47.8 & $-8.16 \%$ & -17.6 & -19.6 & $-11.36 \%$ \\
\hline
\end{tabular}

As shown in Table 3, the prediction error of flow volume $\Delta V_{t w}$ and $\Delta V_{b w}$, between the measured results and the analytical results, are not greater than $\pm 16 \%$. The prediction error maybe be caused by following reasons, such as the measured error for the cross-section profile of outgoing workpiece is not regular and difficult to be measured, the measuring and calculating error in getting the cross-section size and the volume of outgoing workpiece, the prediction error of spread coefficient $\beta_{t}$, $\beta_{b}$ for the spread prediction model was derived by modifying the existing empirical formula. 


\section{Conclusions}

(1) The elongation coefficient $\lambda_{w}, \lambda_{t}$ and $\lambda_{b}$ are all proportional to the reduction coefficient $\eta_{w}$, and they all inversely proportional to the integrated deformation influence factor $\beta_{b} * \eta_{w} / \eta_{b}$ and $\beta_{t} * \eta_{w} / \eta_{t}$.

(2) The deformation parameters of the head of rail $\beta_{t}^{*} \eta_{w} / \eta_{t}$ has no remarkable influence on the elongation coefficient $\lambda_{b}$, and the deformation parameters of the base of rail $\beta_{b} * \eta_{w} / \eta_{b}$ has no remarkable influence on the elongation coefficient $\lambda_{t}$.

(3) With the rise of the integrated deformation influencing factors $\beta_{t}^{*} \eta_{w} / \eta_{t}$ and $\beta_{b} * \eta_{w} / \eta_{b}$, the quantity and direction of volume flow between different parts of rail changes obviously.

\section{References}

[1] Xiong Shangwu, Zheng Guofeng, Liu Xianghua, Three-Dimensional Thermo-Mechanical Finite Element Simulation of the Vertical-Horizontal Rolling Process, J. Journal of Materials Processing Technology. 110 (2001) 89-97.

[2] Jin Xiaoguang, Liu Yuli, Lian Jiachuang, Three-dimensional analysis of the universal beam tandem rolling process, J. Journal of Materials Processing Technology. 102 (2000) 65-69.

[3] Jin Xiaoguang, Liu Yuli, Lian Jiachuang, Three-dimensional analysis of the universal beam tandem rolling process, J. Journal of Materials Processing Technology. 102 (2000) 59-64.

[4] M. Glowacki, The mathematical modelling of thermo-mechanical processing of steel during multi-pass shape rolling, J. Journal of Materials Processing Technology. 168 (2005) 336-343.

[5] S.H. Jeong, S.H. Lee, Computer simulation of U-channel for under-rail roll forming using rigid-plastic finite element methods, J. Journal of Materials Processing Technology. 201 (2008) 118-122.

[6] Zong Wei, Gao Michao, Chen Lin, Experimental research on the relation between reductions and the flange length in heavy rail universal rolling, J. Journal of Inner Mongolia University of Science and Technology. 27 (2008) 316-318.

[7] Jin Liang, Yu Chibin, Ye Chuanlong, Mathematical model of defromation calculation in heavy rail universal continuous rolling and its application, J. Special Steel. 30 (2009) 7-9.

[8] Guo Yujing, Xie Zhijiang, Study on Optimization Model of Rolling Parameters of High Speed Rail by Universal Mill, J. hinese Mechanical Engineering. 21 (2010) 1200-1202.

[9] Guo Yujing, Xie Zhijiang, Multiplex analytical method for metal three-dimensional flow of heavy rail rolling by universal mill, J. Journal of Chongqing University (Natural science edition). 33 (2010) $38-42$.

[10] Kosuke Nakashima, Characteristic of H-beam Universal rolling(translated by Sheng Guangxian), J. Foreign Heavy Machinery (Special Edition for section rolling). 1 (1983) 80-86.

[11] Dong Yonggang, Zhang Wenzhi, Theoretical and Experimental Research on the Spread of the Head of Rail in Rail Rolling by a Universal Mill, J. Chinese Mechanical Engineering. 20 (2009) 1004-1007

[12] Dong Yonggang, Zhang Wenzhi, Theoretical and experimental research on spread of base of rail in universal rolling, J. Journal of Central South University (Science and Technology). 42 (2011) 2676-2680. 